\title{
DEVELOPING AN AUTOMATION SYSTEM FOR GENERATING THE CAD MODEL OF SPUR GEAR
}

\author{
JAYAKIRAN REDDY $\mathbf{E}^{\mathbf{1}}$, RAJENDRA $\mathbf{D}^{\mathbf{2}} \boldsymbol{\&}$ VENKATESU NAIKJ $\mathbf{3}^{\mathbf{3}}$ \\ ${ }^{I}$ Department of Mechanical Engineering, Sreenidhi Institute of Science \& Technology, Hyderabad, Telangana, India \\ ${ }^{2,3}$ Department of Mechanical Engineering, School of Engineering and Technology, Sri Padmavati Mahila Visvavidyalayam,
}

Tirupati, Andhra Pradesh, India

ABSTRACT
Traditional CAD modeling approaches of spurgear are time consuming as the geometry is complex. A small change in
the shape or size of the spurgear in a transmission system will cause a significant change in the dimensions of it because
of the interrelated parts. As a result, the CAD models of the spurgear need to bedone again with modified dimensions.
The modeling technique like parametric modeling technique offers the solution to the issues like these. This paper
addresses the issue like this and offers the solution for the quick CAD model generation. The projected solution is the
development of KBS for the CAD model generation of the spurgear. The developed frameworkis a preliminary system
for the engineering application which utilizes the SolidWorks software and uses the reuse of the design knowledge.
KEYWORDS: Spur gear; Parametric Modeling; SolidWorks API; Macro; KBS \& Automation

Received: Jun 06, 2020; Accepted: Jun 26, 2020; Published: Aug 19, 2020; Paper Id.: IJMPERDJUN2020806

\section{INTRODUCTION}

Spur Gearis one of the important parts in any mechanical transmission system where power need to betransferred though the shafts. Though the design process of spurgearhas been standardized, its CAD models need to be generated manually and itis time consumingtask. If any change needs to bedone in the design requirements of the existing part then the whole design need to bedoneagainfrom the scratch as itmay cause changes in the chained parts. Hence, itis a time-consumingtask. So, there shouldbe some design alternative for developing the CAD model of the spurgear. The advance dmodeling techniques in CAD like parametric modeling technique offers solution to this issue. As everyone knows $\mathrm{CAD}$ is an important and unavoidableprocess in the design practices. In order to reduce the product design time, manyresearchersproposed to use advanced tools like KBS. Thoughitis a developing discipline, its most dramatic impact wouldbe the automated generation of a KBE application basing on general knowledge. This paper proposes a KBS for developing the CAD model of the spurgear to reduce CAD modeling time.

CAD (Computer Aided Design) is a tool which is extensively used in many applications, including automotive, ship building, and aerospace industries, industrial and architectural design, and many more. CAD is also widely used for detailed engineering process of 3D models and/or 2D drawings of physical components, but it is also used throughout engineering process. So, theyneed to becustomized for improving their effectiveness. However, modeling is a time-consuming process because of complexity in design and lack of skilled CAD modeling professionals in market [1]. The companies are looking for the alternate methods for designing the product. There is a need to customize and improve CAD/CAM effectiveness [2]. The production of small volume with diverse product types is the new trend. In order to achieve these needs in less time a Knowledge Base system for engineering and designing is required [3]. By parametric modeling technique one can regenerate the task in veryless time in comparison with 
human [4]. The parametric modeling technique is dimension driven and it facilitates automaticre-use of existing design processbased on the results of engineering analysis [5].

The spurgear is the simplest type of gear having teeth projecting radially. Generally, the efficiency of gearisnearly 98\%. Recently, commercial CAD tools are preferred by the designers to design the gears. Modeling the gearusing CAD not only helps in drawing the complex teeth curves but also makes it easy to manufacture the gear as the CAD model is the input to the presentday CNC machine. But, generating and regenerating the geometries of gear is complexusing CAD software. Additionally, gear CAD modelingis a time-consuming process because of lack of CAD modeling professionals [1].

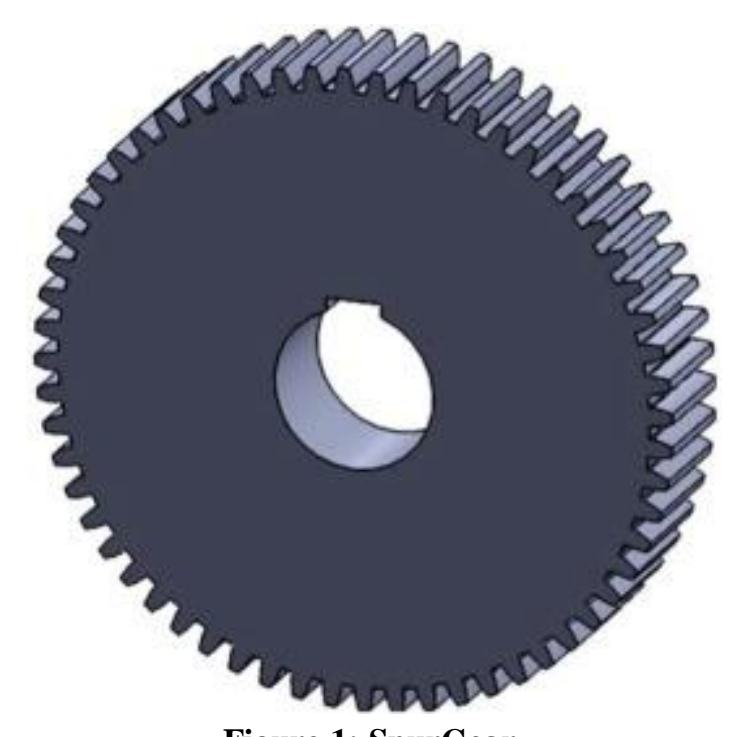

Figure 1: SpurGear.

\subsection{Knowledge Based Engineering (KBE)}

$\mathrm{KBE}$ is an engineering method that represents a merging of Artificial Intelligence (AI) techniques and CAD technologies, giving benefit to customized or variant design automation solutions [6]. The KBE systems aim to capture product and process information. To model engineering design process and then use the model to automate all or part of the process. The product model is an internal computer representation of the product design process. It contains information on both the product and processes that creates the part. The emphasis is on providing, informational complete product representations, captured in a product model. The ultimate goal of the KBE system should be to capture the best design practices and engineering expertise into a corporate knowledge base [6].

\subsection{SolidWorks API}

Application Programming Interface (API) is a tool which provides integration between different applications. It is done by providing a code in a programming language within another application. The API contains hundreds of functions that you can call from Visual Basic for Applications (VBA), VB.NET, Visual C\#, Visual C++ 6.0, and Visual C++/CLI. These functions provide direct access to SolidWorks functionality such as creating a line, inserting an existing part into a part document or verifying the parameters of a surface. With the help of SolidWorks API the automation of modeling, assembling of components is possible by writing specific codes. SolidWorks API is a tree like hierarchical structure which covers all the functions of the software [4].

\subsection{Visual Basic Language}


Visual Basic is a third-generation programming language first released by Microsoft in 1991. It is user-friendly programming language designed for beginners, and it enables anyone to develop GUI (Graphical User Interface) windows applications easily. Additionally, VB is a successful engine for generating the macros in all Microsoft software. For this reason, VB has become a useful tool for generating different programming codes for many applications like SolidWorks. With the help of ActiveX DLL (ActiveX dynamic link libraries) programmers can integrate VB with different windows applications. And it can develop new menus, tools and toolbars into the application environment.

\subsection{Macros}

In computer science macro stands for rule or pattern that specifies how a certain input sequence should be mapped to an output sequence according to a defined procedure. The interaction with the model environment is important to improve the design automation process. CAD systems are providing interfaces via API, which enables the user to interact with the model environment with the help of macros [7]. Also, with the increasing need for sharing data across applications from various disciplines to provide solutions for real-world problems. In many cases there is also the need to implement specific algorithm to perform an accurate computation which cannot be found in any commercial software [1]. These are the main motivations to use API within commercial software. Further, it allows generating geometrical entities and operations with computational algorithm.

\section{Reason to Write the Macro $[6,8]$}

- If a particular operation is always done in the same way, every time, the MACRO automates common and repetitive tasks by commanding the programmed software to do such tasks.

- Design optimization: With use of a few inputs from the user, a Macro can automate the design process weather 2D or 3D designing. Likewise, in general purpose 3D CAD packages Macro can create and analyze design iterations and output the data of each variation.

- Macro can form the basis for the development of special tools to help better visualization of the CAD designs.

- Good Macro programs can consistently retrieve or export data that already exists in the CAD solid modeler's documents.

- Further Macro increases productivity, saves time, and helps to standardize procedures.

\subsection{Parametric Modeling Technique}

Parametric modeling technique is one of the sophisticated CAD modeling approaches that give out the traditional 2D approaches. It allows the reuse of the already existing procedures to carryout the quick design changes based on the existing rules of the engineering $[1,5]$.

\section{LITERATURE REVIEW}

For accurate and effortless solid modeling one can use macro programming along with parametric modeling technique [1]. With these tools, the generation of CAD model of the spur gear can be done as these tools are capable of simplifying the complexities which arise while modeling. Further, with these tools automation of the CAD modeling is also possible especially the frequently executing tasks can be automated with a predefined algorithm [9].

In the process of reducing the CAD modeling time, Bo et al. [10] developed a library by using SolidWorks which 
contains the general standard parts. Similarly, to generate the CAD model through automation, Wei et al. [11] proposed an approach on SolidWorks platform. In the same way, Myung et al. [12] projected an approach for modeling the machine tool using parametric modeling technique. Using this technique, Myung et al. were able to minimize the CAD modeling time. Jayakiran et al. [13] developed a framework on SolidWorks platform which uses knowledge based system for CAD modeling and manufacturing. By using SolidWorks, automation of CAD modeling is possible with the help of API and macro [1].

Knowledge based system is a computer software involves maximizing probability of developing sustainable software within limitations. In knowledge based system, one can perform analysis, design and implementation phases simultaneously and repeatedly in short cycles [14].

Though much work was carried out on development of the parametric modeling of components, no work was targeted on spur gear. Hence, authors believed that a dedicated automatic CAD modeling system for generating a spur gear is required for the present market and research world as the spur gear is an important part in any energy transmission systems through shaft.

At present, most of the commercial CAD software are targeted to develop the part model of the component by conventional approached and by accepting the input data in the text format related to the model. Besides, for feeding this text input, a computer program can also be used [1]. This paper adopted this approach for feeding the input data. This program can be written using the specific programming languages such as VBA, C++, Lisp etc. [2]. In these languages, VBA is considered as the convenient one. The SolidWorks CAD software uses VBA as programming editor. Hence, the authors preferred to choose SolidWorks as CAD software for developing the proposed system to generate the automatic CAD model of the universal coupling. Additionally, macro code is also used for generation of the CAD model of the spur gear as it facilitates the automatic generation of the CAD model.

\section{METHODOLOGY FOR MODELING AND AUTOMATION}

At present, most of the commercial CAD software are targeted to develop the part model of the component by conventional approached and by accepting the input data in the text format related to the model. Besides, for feeding this text input, a computer program can also be used [1]. This paper adopted this approach for feeding the input data. This program can be written using the specific programming languages such as VBA, C++, Lisp etc. [2]. In these languages, VBA is considered as the convenient one. The SolidWorks CAD software uses VBA as programming editor. Hence, the authors preferred to choose SolidWorks as CAD software for developing the proposed system to generate the automatic CAD model of the universal coupling. Additionally, macro code is also used for generation of the CAD model of the spur gear as it facilitates the automatic generation of the CAD model. 


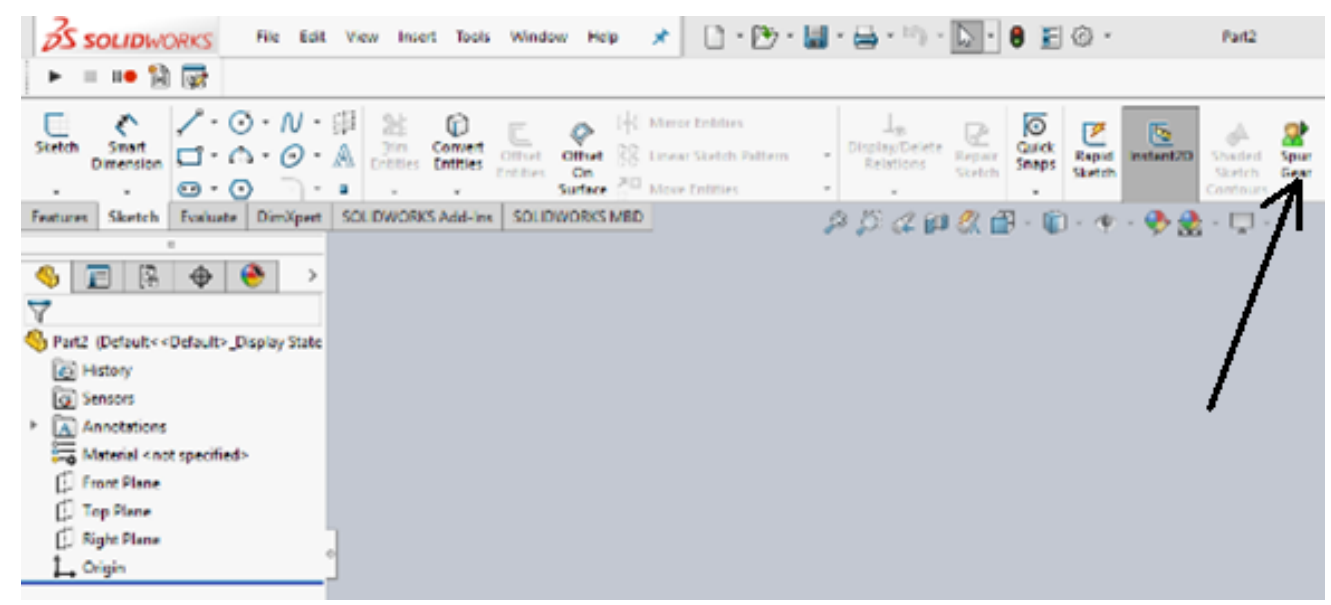

Figure 2: Button for Spur Gear.

For enabling the user to use the proposed system, a button for generating the CAD model of the spur gear was incorporated in the SolidWorks environment. This button is shown in the figure 2.

For collecting the input data from the user, a GUI was developed. Figure3 shows the GUI for generating the spur gear. This GUI was developed using VB which is available in SolidWorks.

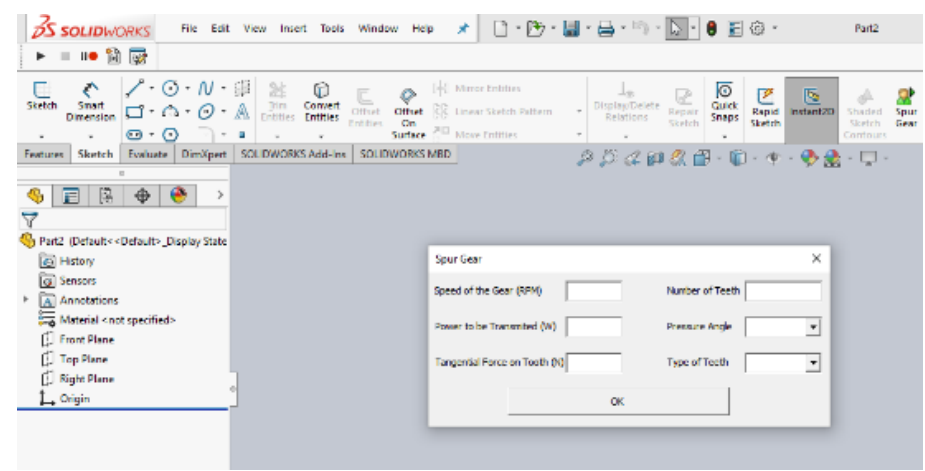

Figure 3: GUI for Universal Coupling.

\subsection{Phases in Developing KBS}

Phase I: Project initialization

Phase II: System analysis and design

Phase III: Rapid prototyping

Phase IV: System development

Phase V: Implementation.

\section{Project Initialization}

In every process project initialization is primary and important phase. The project initialization is stage set for development of a new system. The Project initiation involves problem definition, verification for expert approach and feasibility study.

Problem definition: Problem definition is simplification and involves addressing some essential inquiries like what precisely the issue is, what are the genuine needs, regardless of whether the area of the issue is restricted or not and so on.

Verification for expert approach: Verification for expert approach stage comprises of confirming whether the expert 
and information are accessible; and confirming the interfacing between the expert's knowledge and information is conceivable or not.

Feasibility study: Feasibility study for any KBS is equivalent to for any data-based system. It includes considering the information available and specialized achievability. The technical possibility question includes whether the technology exists to build the system.

\section{System Analysis and Design}

This phase involves detailed system analysis to estimate system's performance. This involves tasks Conceptual design, Development strategy, Source of knowledge.

Conceptual design: It provides a general idea of what the system will resemble and how it will solve the problem.

Development strategy: Development strategy must offer the response for the inquiries like how to arrive at the solution of the project, the most ideal approach to take care of the problem.

Source of knowledge: KBS utilize both human expertise and recorded sources for the information. The more human skill required the more drawn out and progressively entangled the procurement procedure becomes. The entire thought on KBS is testing, so an accomplished expert required.

\section{Rapid Prototyping}

A prototyping in KBS starts as a small-scale system. It includes representation of the knowledge captured in a manner that enables quick interfacing and creation of the major components of a KBS on a fundamental basis.

\section{System Development}

It involves continuous testing, review and improvements. A user interface is created for database interaction. Here we evaluate KBS comparing its performance with accepted values till we get the satisfactory results. In order to develop a robust KBS this process must be done like iterations.

\section{Implementation}

This process can be long and complex, it consists acceptance by user, security issues etc.

Acceptance by user: It depends on the quality and ease of use because of behavioral and psychological considerations varies from user to user.

Security issues: to protect developed software from unexpected crashes.

\subsection{Generating the Dimension Specific Model Through Macros}

To generate model of the component required, the sample component is first modeled completely in SolidWorks and macro is recorded for it. The dimensions of the component can be varied accordingly and the macro is run, thus various component models are generated.

\subsection{KBS for Spur Gear}

For generating the model, a separate macro was developed for the spur gear. The inputs for the model are standard dimensions of spur gear like addendum circle diameter, pitch circle diameter, base circle diameter, dedendum circle diameter. 


\section{RESULTS AND DISCUSSIONS}

This section presents the sample result that was generated by the proposed KBS. The sample input data for the generation of the spur gear areconsidered as addendum circle diameter $=493 \mathrm{~mm}$, pitch circle diameter $=477 \mathrm{~mm}$, base circle diameter $=$ $448 \mathrm{~mm}$, dedendum circle diameter $=457 \mathrm{~mm}$. Using this sample data, the CAD models of the spur gear is generated by the using the proposed KBS which is shown in the figure 4.

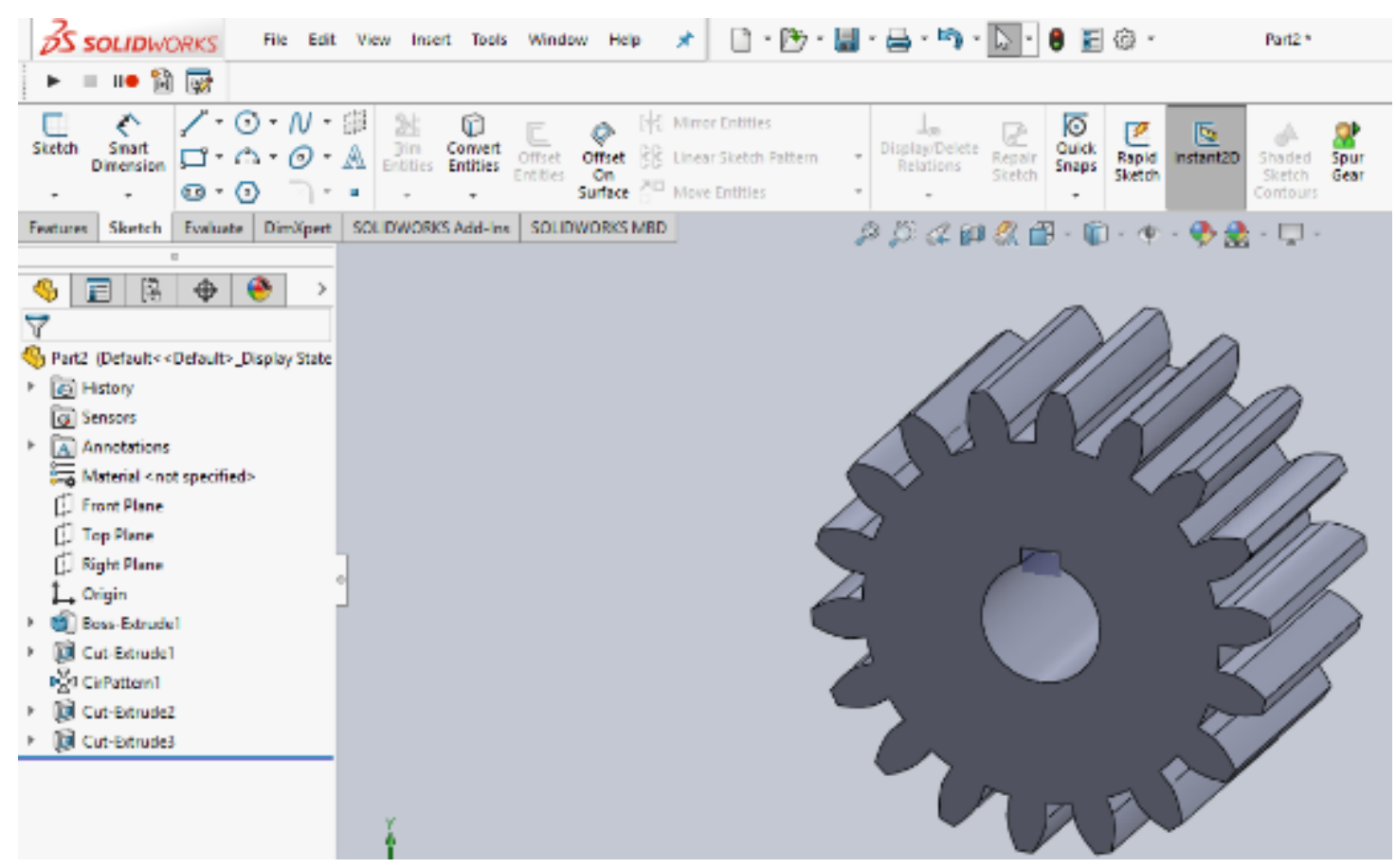

Figure 4: Spur Gear.

On comparing these $\mathrm{CAD}$ models with the $\mathrm{CAD}$ models which are generated using the traditional modeling process, it is found that they are very close in dimensions. Moreover, it is found that the time taken for the generating the complete CAD model of the universal coupling using the proposed system is 21 seconds, whereas the time taken for the developing the CAD model of the universal coupling using the traditional process is the 1860 seconds.

\section{CONCLUSIONS}

This paper proposes a KBS for generating the CAD model of the Spur Gear. For generation, the proposed system uses parametric modeling technique and utilizes the reuse of the design process. The system is user friendly and handy to use at any computer which is having the SolidWorks installed in it. The system has proven itself as the faster than the traditional approach for generating the CAD model of the universal coupling.

In future, the same system can be further improved to do analysis of the modeled component by incorporating it into any CAE software.

\section{REFERENCES}

1. E Jayakiran Reddy, V Pandu Rangadu(2018), "Development of Knowledge Based Parametric CAD Modeling system for Spur 
Gear: An Approach", Alexandria Engineering Journal, Vol. 57, pp. 3139-3149.

2. Jayakiran Reddy E, Sridhar C N V, Pandu Rangadu V (2016), "Development of KBS for CAD modeling of Industrial Battery Stack and its Configuration: An approach", Advances in Intelligent Systems and Computing, Vol. 530, pp. 607-618.

3. Jayakiran Reddy E, Sridhar C N V, Pandu Rangadu V (2016), "Development of KBS for CAD modeling of a two wheeler IC Engine Connecting Rod: An approach", Advances in Intelligent Systems and Computing, Vol. 530, pp. 597-606.

4. Poluru, Suswanth, and VijayPandey. "Improving the BendingStrength of SpurGearTooththrough Profile Modification usingCad." International Journal of Mechanical and Production Engineering Research and Development (IJMPERD) 7.4 (2017): 39-48.

5. Jayakiran Reddy E, Sridhar C N V, Pandu Rangadu V (2016), "Research and Development of Knowledge Based Intelligent Design System for Bearings Library Construction Using SolidWorks API", Advances in Intelligent Systems and Computing, Vol. 385, pp. 311-319.

6. E Jayakiran Reddy, N Venkatachalapathi, V Pandu Rangadu (2018), "Development of an approach for Knowledge-Based System for CAD modelling", Materials Today: Proceedings, Vol. 5, pp. 13375-13382.

7. Ngo, Minh Tuan, and Vinh Sinh Hoang. "A method for reducing the tooth profile errors of the spurgears in the hobbingprocess." Int J MechProd Eng Res Dev 9 (2019): 1-10.

8. E Jayakiran Reddy, CN V Sridhar, V Pandu Rangadu (2015), "Knowledge Based Engineering: Notion, Approaches and Future Trends", American Journal of Intelligent Systems, Vol. 5, pp. 1-17.

9. E Jayakiran Reddy, V Pandu Rangadu, R Ramachandra (2018), V Naga Prasad Naidu, "Development of a constraint-based approach for Knowledge-Based System for CAD modeling", Advanced Science, Engineering and Medicine, Vol. 10, pp. 357361 .

10. Bernard, Mushirabwoba, et al. "Preliminary Study Of Frictional Power Losses In SpurGeared Transmissions. "International Journal 7 (2017): 199-208.

11. E Jayakiran Reddy, C N V Sridhar, V Pandu Rangadu (2015), "Knowledge - Based Parametric Modeling for Bolts, Nuts and Bearings using SolidWorks", International Journal of Applied Engineering Research, Vol. 10, pp. 16111-16120.

12. E. Jayakiran Reddy, R. Rama Chandra (2017), "Development of an Intelligent System for the Design of a Two Wheeler IC Engine Connecting Rod", International Journal of Engineering Science Invention, Vol. 6, pp. 67-74.

13. Aru, Suraj, et al. "Design and Analysis of Centrally Suspended Cage-Less Differential." International Journal of Mechanical and Production Engineering Research and Development (IJMPERD) 4.4 (2014): 49-60.

14. Bo Sun, Guangtai Qin, Yadong Fang (2011), "Research of Standard Parts Library Construction for SolidWorks by Visual Basic", In: International Conference on Electronic \& Mechanical Engineering and Information Technology, pp. 2651-2654.

15. Wei Liu, Xionghui Zhou, QiangNiu, Yunhao Ni (2014), “A Convenient Part Library Based On SolidWorks Platform”, International Journal of Mechanical, Aerospace, Industrial and Mechatronics Engineering, Vol. 8, No. 12, pp. 1851-1854.

16. Sehyun Myung, Soonhung Han (2001), "Knowledge-based parametric design of mechanical products based on configuration design method", Expert Systems with Applications, vol. 21,pp. 99-107.

17. E. Jayakiran Reddy, R. Rama Chandra (2017), "Development of an Intelligent System for the Design of a Two Wheeler IC Engine Connecting Rod", International Journal of Engineering Science Invention, Vol. 6, pp. 67-74. 
18. E Jayakiran Reddy, J VenkatesuNaik, D Rajendra, V Pandu Rangadu (2020), "Online Knowledge-Based System for CAD modeling and Manufacturing - An Approach", Advances in Intelligent Systems and Computing, Vol. 910, pp. 259-268. 
